\title{
Refractory Head and Neck Squamous Cell Carcinoma
}

National Cancer Institute

\section{Source}

National Cancer Institute. Refractory Head and Neck Squamous Cell Carcinoma. NCI

Thesaurus. Code C148152.

A head and neck squamous cell carcinoma that does not respond to treatment. 